\title{
Prognostic Evaluation of Cerebral Venous Sinus Thrombosis Using Clinical and Mr Sequences
}

Suvendu Mohapatra ${ }^{1 *}$, Basant Manjari Swain ${ }^{1}$ and Jayashree Mohanty ${ }^{1}$

${ }^{1}$ Department of Radiology S.C.B Medical College, Cuttack, Odisha, India

*Corresponding author: Dr Suvendu kumar Mohapatra M.D, Department of Radiology, S.C.B Medical College \& Hospital, Cuttack, Odisha, 753007, India, Tel: +09861412800; Fax: +916742390674; E-mail: drsuvendumohapatra@gmail.com

Received date: Apr 22, 2014, Accepted date: Aug 12, 2014, Published date: Aug 17, 2014

Copyright: (c) 2014 Mohapatra S, et al. This is an open-access article distributed under the terms of the Creative Commons Attribution License, which permits unrestricted use, distribution, and reproduction in any medium, provided the original author and source are credited.

\begin{abstract}
Due to diverse clinical presentation of cerebral venous thrombosis it is often under diagnosed in clinical scenarios first diagnosed by radiologist. Present study is targeted to identify etiology, risk factors, clinical presentation, radiological findings and its prognostic importance in patients with cerebral venous thrombosis (CVT).
\end{abstract}

Materials and methods: Patients age ranging from 14 to 68 years with clinical suspicion of cerebral venous thrombosis was subjected to CT, MRI scan. Patients with positive radiological findings are followed upto 8 weeks.

Results: in our study we have concluded that;

1. CVT incidence is not uncommon in males,

2. Though puerperium is a high risk factor for cerebral venous thrombosis, it is not always associated with poor outcome.

3. Presence of altered sensorium and focal neurological deficits at admission predicts poor outcome.

4. CT is a good first line investigation for CVT,

5. Features predictive of poor clinical outcome are Presence of parenchymal hypodense lesions in CT, deep parenchymal T2 hyperintense lesion, restriction of diffusion. Presence of altered sensorium and focal neurological deficit at admission.

Keywords: Cerebral venous thrombosis; Prognostic; Magnetic resonance imaging; Diffusion weighted imaging

\section{Introduction}

\section{Etiology}

The cerebral venous system consists of dural venous sinuses, superficial cortical veins, deep medullary and subependymal veins [1]. Cerebral veins contain about $70 \%$ of the total cerebral blood volume but CVT occurs about thousand times less often than arterial stroke. Arterial and venous stroke cause different neurological deficits and occur in people of different ages. About half of the patients with an arterial stroke are older than 75 years, whereas CVT most often affects young adults and children. Any pathology that alters the Virchow's triads of flow vessel wall and fluid blood physiology can cause thrombosis [2]. Stroke in young adults accounts 30\% of all cases. Among this $10 \%$ to $20 \%$ are due to CVT [3]. Thrombosis of the cerebral veins may cause focal deficits due to local effects of venous obstruction but also more generalized effects as a result of increased cerebrospinal fluid pressure caused by blocking of the major sinuses. In the majority of patients these two processes occur simultaneously. The course and clinical features of CVT are highly variable. The various causes [4] are Idiopathic, Pregnancy and Puerperium, mastoiditis, dehydration, Oral Contraceptive Pills, Local tumor extension, Trauma, Coagulopathies-Antiphospholipid syndrome, Antithrombin III deficiency, Factor V Leiden mutation, Protein C/S deficiency, PNH, Inflammatory bowel disease.

\section{Clinical features}

CVT is protean in its presentation making it very difficult to diagnose unless a high degree of suspicion is present. The presentation can be acute subacute, or chronic. The various signs and symptoms at presentation are [5]. Headache-Most common, present in $>80 \%$ of cases. Seizures-Present in 35\%- $40 \%$ of cases. It can be focal or generalized. Focal neurological signs-30\%-35\%. Altered sensorium-30\% Vomitting.

The signs and symptoms of CVT may mimic arterial stroke, intracranial tumors, intracranial infection, subarachnoid hemorrhage or psychiatric disorders. The mortality rate ranges from $10 \%$ to $15 \%$. The mortality is usually in the acute stage. The cause being involvement of brainstem, deep parenchyma, transtentorial herniation due to mass effect. The residual deficit rate lies between $6 \%$ to $20 \%$. The focal neurological deficits at admission may or may not be reversible. Simple edema and venous congestion, compression due to 
mass effect may reverse with treatment. Venous infarcts parenchymal hematoma and secondary ischemic necrosis results in permanent neuronal damage and deficits. Depending on the region of brain involvement the deficits can be hemiparesis, monoparesis, paraparesis, blindness, cranial nerve palsy or dysphasia.

\section{Diagnosis}

Venous thrombosis has a nonspecific presentation and therefore it is important to recognize subtle imaging findings and indirect signs that may indicate the presence of thrombosis as CVT mostly unsuspected by clinician's radiological diagnosis remains an important part in its management.

The various modes of investigations are 1.CT scan .2.MRI. 3.Catheter Angiogram. 4. Transcranial Doppler 5.biochemical

\section{Computed tomography}

The first investigation to be undergone by a patient with CNS signs and symptoms is CT. CT helps in diagnosing $>80 \%$. CT plays an important role in ruling out intracranial abscess or tumors. The various signs of CVT in CT are: Hyperdense triangle sign-Cord sign, Empty Delta sign, Parenchymal hemorrhage, Parenchymal hypodensity. False positive empty delta sign is seen in high splitting tentorium, subdural hematoma, subdural empyema. Recently using MDCT venogram of intracranial venous system can be acquired [4].

\section{Catheter angiogram}

The thrombosed sinus appears as empty channel devoid of contrast surrounded by collaterals. Thrombosed cortical veins are seen as intraluminal filling defects and in delayed phase as cord of contrast collection. Since CT and MRI are sensitive and specific in CVT need of catheter angiogram is obviated [6].

\section{MRI}

T1WI, T2WI, FLAIR, T2*WI MRV and DWI are used in diagnosis of sinus thrombosis and the parenchymal changes. 2D TOF MRA technique is used for venogram of cerebral sinuses. The thrombus within the sinus can be imaged with T1 and T2 weighted images or with MRV. In T1 and T2 weighted images the normal venous sinus shows flow void due to moving blood. In thrombosed sinuses the flow void is absent with varying signal depending on the age of thrombus and the sequences used. Absent signal with frayed appearance is a feature of thrombosis. Hypoplastic venous sinus, in plane effect, slow flow is some artefactual conditions for false positive finding. The parenchymal edema appears hyperintense in T2WI and FLAIR images. Venous infarcts appear hyperintense in T2 WI and FLAIR images. It is differentiated from edema by DWI which shows hyperintense regions. Hemorrhage can be intraparenchymal or in subarachnoid space. Intraparenchymal hemorrhage appearance in T1 and T2 images vary with the stage of hemoglobin breakdown. FLAIR sequence is highly sensitive for subarachnoid hemorrhage [7].

\section{Transcranial Doppler}

Ultrasound techniques may have a role in diagnosing CVT [8].

\section{Biochemical investigations}

Quantitative analysis of $\mathrm{D}$ - dimer in blood is done. This is a breakdown product of the thrombus. A measurement of $>500 \mathrm{ng} / \mathrm{ml}$ is highly suggestive of CVT. It has a sensitivity of $83 \%$ and specificity of $90 \%$ [9]. It is a useful screening modality.

\section{Treatment}

The management of CVT consists of symptomatic treatment and definitive treatment of the thrombus. It consists of antiepileptics, treatment of raised intracranial hypertension, antithrombotics, treatment of primary cause, recanalisation of thrombotic veins.

Seizure is a poor prognostic factor. It results in secondary ischemia and brain damage. So control of seizures using antiepileptics both oral and parenteral remain one of the mainstay in CVT treatment.

CVT results in venous congestion, edema, hemorrhage and infarcts. These results in raised ICT. This can result in transtentorial herniation of brain parenchyma and brainstem compression leading to sudden death. So prompt treatment of raised ICT prevents much of the mortality associated with CVT. Treatment consists of medical and surgical methods. Medical management includes IV mannitol. Surgical treatment includes burrhole decompression of cranium, hemicraniectomy, surgical debridement [10].

Heparin remains the firstline treatment of CVT [11] Recent studies show its safety even in hemorrhagic lesions. It is followed by oral anticoagulants for atleast 12 months.

Local thrombolysis is reserved for those who donot respond well to the above treatment. Local urokinase infusion into the thrombosed sinus results in lysis of thrombus. Complication include increased intracranial bleed and pelvic bleed from the puncture site.

\section{Aims and objectives}

A comprehensive study was conducted to identify the significance of various etiology, risk factors, clinical presentation, radiological findings and its prognostic importance in patients with cerebral venous thrombosis (CVT).

\section{Materials and methods}

Out study is a prospective cohort study of 36 patients age ranging from 14 to 68 carried out at Department of Radiology, S.C.B Medical College, Cuttack between 2009-2011. All the 36 patients were subjected to CT scan and MRI. MRI was performed using GE Signa HDX MR machine with a 1.5 tesla field strength magnet. For MR venogram 2D TOF protocol was used Precontrast images were taken followed by postcontrast images with intravenous administration of $0.1 \mathrm{mmol} / \mathrm{kg}$ of body weight of gadolinium. The standard imaging protocol was used.

\section{Study design and statistical method}

It was a Prospective cohort study. Null hypothesis testing was done by students t-test using SPSS software 2010 version.

\section{Study population and Sample size:}

36 patients with radiologically proven CVT were taken into study and were followed upto 8 weeks. 


\section{Inclusion and exclusion Criteria}

All patients confirmed by MRI and MRV as cerebral venous sinus thrombosis. Both Males and females are included. All age groups included. Patients with MR incompatible devices or implants. Patients on life support systems. Patients with claustrophobia.

\section{Study method}

The clinical features at admission were analysed concentrating on.

Clinical signs: Headache, Seizure, Focal or generalized, Altered sensorium, coma,Focal neurological deficit-Hemiparesis, paraparesis, monoparesis, Cranial nerve palsy, blindness, dysphasia. CT scan findings: presence of hyperdense cord sign, parenchymal haematoma, parenchymal hypodensity. MRI Findings: Hemorrhage, Thrombosis within Sinuses. T2 hyperintense lesion in superficial and deep cerebral regions. Diffusion Restriction-Using DWIs and ADC map.

\section{Results}

In this study done in Dept of Radiology S.C.B Medical College, between September 2009 to dec 2011, 36 patients were included. All these patients were selected with clinical features suggestive of cerebral venous thrombosis with $\mathrm{MR}$ evidence of cerebral venous sinus thrombosis.

The age range of the affected patients is 14 to 68 with a mean age of 41. There is no statistically significant association of age with poor outcome in our study as against the study of Stolz, Rahimi et al. [12] who showed increasing age to be a poor prognostic indicator. The Male: Female sex ratio in this study is 1:1.57 that is, males form $38 \%$ of the patients. This shows that CVT is not uncommon among males and should be a differential diagnosis in any patient with suspicious CNS signs and symptoms. Sex does not play a significant role in outcome of CVT in this study.

In a study by José M. Ferro et al. [13] showed male sex to be a poor prognostic factor for CVT.

Among the 22 female patients 15 were in puerperal period that is $68 \%$ of the female patients. This proves that puerperium is high risk for CVT but the outcome among puerperal females does not show any significant difference from the nonpregnant female $\mathrm{p}$ value $>0.05$. High incidence in peurperium corresponding with $\mathrm{C}$ Cantu and $\mathrm{F}$ Barinagarrementeria et al. [14] which also showed high incidence in same group.In the study of Appenzeller et al. [5] pregnancy and puerperium, were good prognostic indicators.but in this study peurperium is not a very prognostic factor may be due to delayed time of diagnosis and late treatment.

In this study the percentage of presenting symptoms are Headache-77\% Seizure-25\%, Altered Sensorium-33\% Neurological Deficit-17\%. Among these presenting symptoms, altered sensorium and focal neurologic deficit are statistically significant poor prognostic indicators at 8 weeks follow up.

This is in agreement with the study of Appenzeller et al. [5] and Ferro, Canhao et al. [15]

The number of patients with parenchymal hemorrhage detected by CT is 9 , out of which 3 had poor outcome as per table nos.8. It is found that this sign is not a poor prognostic indicator with $\mathrm{P}>0.05$.

According to Ferro, Canhao et al. [15] hemorrhage on admission $\mathrm{CT}$ is associated with poor outcome.
The incidence of thrombosis in CT are Superior Sagittal Sinus-75\%, Lateral Sinus-36\%, Straight Sinus-11\%. There is no significant association of thrombosis with the patient outcome.

The presence of parenchymal hypodensity in CT which can be either simple vasogenic edema or a venous infarct was found to be in 7 patients (19.6\%) of which 3 died and 3 had deficit. This has a highly significant association with poor outcome with $\mathrm{P}<0.001$.

$41.6 \%$ of patients had intraparenchymal hemorrhage detected by MRI. 4 patients out of this had poor outcome. That is 4 out of the 8 poor outcome group had hemorrhage. This is not a statistically significant association.

The presence of thrombosis detected by MRI are Superior Sagital Sinus-89\% Lateral Sinus-75\%, Straight Sinus-20\%.This corresponded with similar statistics in literature.

The presence of thrombosis in superior sagittal sinus, lateral sinus and straight sinus is not associated with poor outcome. Ferro Canhao et al. [15] predicted a poor outcome with deep cerebral venous thrombosis. Deep Cerebral venous thrombosis is associated with thalamic and deep grey matter and brainstem lesion. So it is associated frequently with altered sensorium and poor Glascow coma scale. This explains the poor outcome of patients with deep cerebral venous thrombosis.

Straight sinus thrombosis is seen in 4 patients in CT whereas in MRI in 7 patients showing the greater pickup rate in MRI.

Detection of deep T2 hyperintense lesions has a poor outcome. In our study 8 out of 36 had deep T2 hyperintense lesions. Out of this 8 patients 7 (87.8\%) had poor outcome. In our study of the 8 poor outcome patients 7 (87.8\%) had deep T2 hyperintense lesions. So deep hyperintense lesion are sensitive and specific in predicting poor outcome with $\mathrm{p}<0.001$ showing a strong association. In a study by Imada et al. [16] which shows similar association.

Edema can be vasogenic or cytotoxic edema. Cytotoxic edema is due to entry of water into the cells due to decreased ATP. This in course leads to cell death and infarction. So cytotoxic edema is the precursor of infarction. Since intracellular macromolecules and membranous organelles interact and bound with the intracellular water molecules, these protons have restricted diffusion. This can be detected by using DWI in which separate diffusion gradients of varying strengths are given and the signal loss due to diffusion is measured. In DWI free protons have decreased signals in higher gradients, whereas bound or restricted protons give higher signals or hyperintense. It is to be remembered that cytotoxic edema is only the precursor of infarction. In our study group all the 8 patients who had poor outcome had restricted diffusion.

In other words all poor outcome patients had diffusion restriction i.e $100 \%$ sensitive in predicting outcome. But none of the patients who had restricted diffusion recovered completely. According to Mullins et al. [17] three out of 14 lesions had restricted diffusion but resolved in follow-up and 4 out of 14 had restricted diffusion and persisted. In our study against the above study 8 out of 10 had restricted diffusion and had poor out come and none out of 10 had restricted diffusion and with good outcome. Reversibility of DWI restricted lesions are shown in the studies of Appenhium et al. [18], Farbe et al. [19]. 
Citation: Mohapatra S, Swain BM, Mohanty J (2014) Prognostic Evaluation of Cerebral Venous Sinus Thrombosis Using Clinical and Mr

\section{Conclusion}

In our study of conclusions can be summarised as follows. High degree of clinical suspicion required for diagnosis of CVT because of its varied nonspecific clinical features. CVT incidence is not uncommon in males. Though puerperium is a high risk factor for cerebral venous thrombosis, it is not always associated with poor outcome. Presence of altered sensorium and focal neurological deficits at admission predicts poor outcome. CT is a good first line investigation for Cerebral venous thrombosis. Presence of parenchymal hypodense lesions in CT is associated with poor outcome and MRI features predictive of poor outcome are deep parenchymal T2 hyper intense lesion and restriction of diffusion.

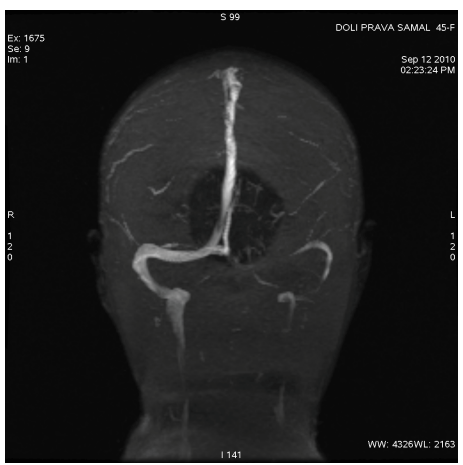

Figure 1: 3D TOF MR Venogram Showing Hypoplastic Left Lateral Sinus

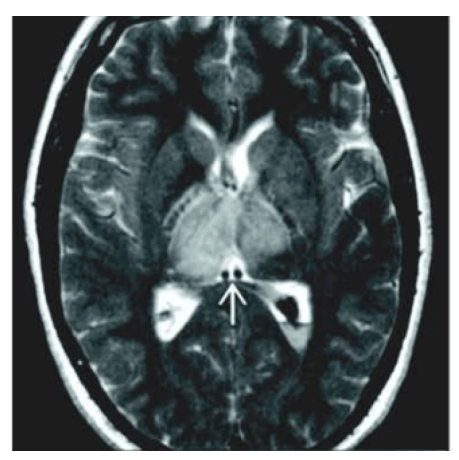

Figure 2: MRI Axial T2 Image Showing Bithalamic Hyperintensity (INFARCT)

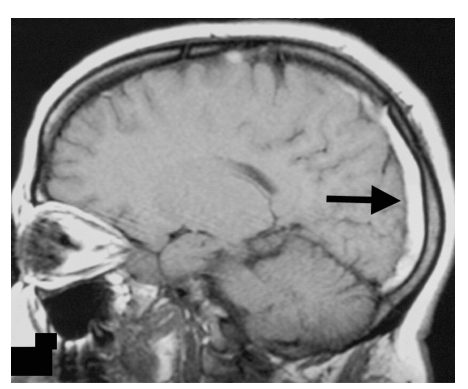

Figure 3: MRI Sagital T1 Showing Superior Sagital Sinus Thrombosis

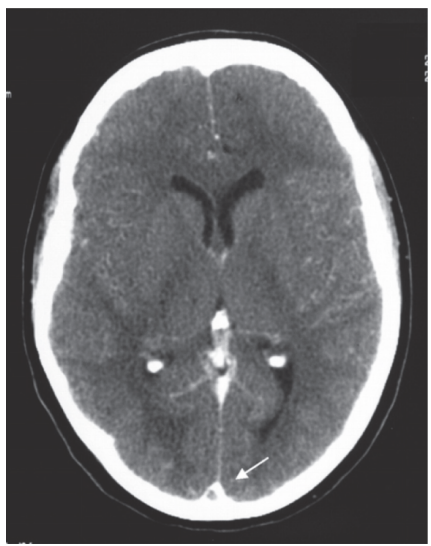

Figure 4: CECT Image Showing Empty Delta Sign

\section{References}

1. Andeweg J (1996) The anatomy of collateral venous flow from the brain and its value in aetiological interpretation of intracranial pathology. Neuroradiology 38: 621-628.

2. van Gijn J (2000) Cerebral venous thrombosis: pathogenesis, presentation and prognosis. J R Soc Med 93: 230-233.

3. Nagaraja D, Sarma GR (2002) Treatment of cerebral sinus/venous thrombosis. Neurol India 50: 114-116.

4. Karthikeyan, Vijay, Kumar, Kanth CVT - Spectrum of CT Findings IJRI $200414: 2: 129-137$

5. Appenzeller S, Zeller CB, Annichino-Bizzachi JM, Costallat LT, DeusSilva L, et al. (2005) Cerebral venous thrombosis: influence of risk factors and imaging findings on prognosis. Clin Neurol Neurosurg 107: 371-378.

6. Lafitte F, Boukobza M, Guichard JP, Hoeffel C, Reizine D, et al. (1997) MRI and MRA for diagnosis and follow-up of cerebral venous thrombosis (CVT). Clin Radiol 52: 672-679.

7. Bakshi, Rohit, Kamran S, Kinkel PR, Bates VE, Mechtler LL, et al. (1999) "Fluid-attenuated inversion-recovery MR imaging in acute and subacute cerebral intraventricular hemorrhage." American journal of neuroradiology 20: 629-636.

8. Caso, Valeria, Giancarlo Agnelli, and Maurizio Paciaroni (2008) eds. Handbook on cerebral venous thrombosis. Karger Medical and Scientific Publishers 23: 50 .

9. Lalive PH, de Moerloose P, Lovblad K, Sarasin FP, Mermillod B, et al. (2003) Is measurement of D-dimer useful in the diagnosis of cerebral venous thrombosis? Neurology 61: 1057-1060. 
Citation: Mohapatra S, Swain BM, Mohanty J (2014) Prognostic Evaluation of Cerebral Venous Sinus Thrombosis Using Clinical and Mr Sequences. J Neurol Neurophysiol 5: 218. doi:10.4172/2155-9562-5-1000218

Page 5 of 5

10. Stam J (2005) Thrombosis of the cerebral veins and sinuses. N Engl J Med 352: 1791-1798.

11. Bousser MG, Ferro JM (2007) Cerebral venous thrombosis: an update. Lancet Neurol 6: 162-170.

12. Stolz E, Rahimi A, Gerriets T, Kraus J, Kaps M (2005) Cerebral venous thrombosis: an all or nothing disease? Prognostic factors and long-term outcome. Clin Neurol Neurosurg 107: 99-107.

13. Ferro JM, Canhão P, Stam J, Bousser MG, Barinagarrementeria F; ISCVT Investigators (2004) Prognosis of cerebral vein and dural sinus thrombosis: results of the International Study on Cerebral Vein and Dural Sinus Thrombosis (ISCVT). Stroke 35: 664-670.

14. Cantu, Carlos, and Fernando Barinagarrementeria (1993) "Cerebral venous thrombosis associated with pregnancy and puerperium. Review of 67 cases." Stroke $24: 1880-1884$.

15. Ferro JM (2002) Cerebral venous thrombosis. J Neuroradiol 29: 231-239.
16. Imada Y, Ikawa F, Kawamoto H, Ohbayashi N, Matushige T, et al. (2004) Usefulness of diffusion-weighted magnetic resonance imaging in diagnosis and therapeutic strategy of deep cerebral venous thrombosis: a case report. No Shinkei Geka 32: 285-289.

17. Mullins ME, Grant PE, Wang B, Gonzalez RG, Schaefer PW (2004) Parenchymal abnormalities associated with cerebral venous sinus thrombosis: assessment with diffusion-weighted MR imaging. AJNR Am J Neuroradiol 25: 1666-1675.

18. Ducreux D, Oppenheim C, Vandamme X, Dormont D, Samson Y, et al (2001) Diffusion-weighted imaging patterns of brain damage associated with cerebral venous thrombosis. AJNR Am J Neuroradiol 22: 261-268.

19. Sarma D, Farb RI, Mikulis DJ, terBrugge KG (2004) Reversal of restricted diffusion in cerebral venous thrombosis: case report. Neuroradiology 46: 118-121. 